\title{
Prix Joseph et Marie-Anne Piller
}

\author{
Julien Knebusch ${ }^{a}$, Alexandre Wenger ${ }^{b}$ \\ ${ }^{a}$ Mer, PhD, Université de Fribourg, chaire Médecine et société; ${ }^{b}$ Po, PhD, Université de Fribourg, chaire Médecine et société
}

Dans le cadre du programme Médecine et société (Medical Humanities) de l'Université de Fribourg, les étudiants en médecine de $3^{\mathrm{e}}$ année effectuent chaque année un exercice obligatoire d'écriture. Après avoir effectué un stage de 4 jours auprès d'un médecin généraliste, ils rédigent le compte-rendu circonstancié d'une rencontre avec un patient qui les a marqués. Leur texte doit répondre à un ensemble de directives formelles insistant sur la cohérence interne du récit, la construction d'ensemble, ou encore la pertinence des informations retenues. Le récit est suivi par quelques lignes qui reprennent la situation décrite, mais sous la forme brève du dossier médical: le contraste entre les deux formes d'écriture doit apparaître clairement. Le Prix Joseph et Marie-Anne Piller récompense le meilleur texte francophone et le meilleur texte germanophone.

Prendre le temps de faire écrire un texte personnel aux étudiants en médecine: l'exercice a de quoi déstabiliser dans un cursus orienté sur le travail collectif, marqué par la course aux connaissances et sanctionné par des QCM.

Pourtant, pour de futurs cliniciens, l'exercice est fondamental. Ecrire, c'est choisir soigneusement ses mots, organiser sa pensée, prendre conscience du destinataire auquel on s'adresse, maîtriser ses propres présupposés. Par l'écriture, l'étudiant appréhende la complexité de la rencontre avec un patient en opérant, par le biais de la narration, une distanciation et un recul réflexif. La lourdeur des études de médecine, l'enchaînement souvent harassant du quotidien médical, rendent nécessaire une telle respiration.

Le langage est une ressource clef pour la communication avec le patient et la pratique clinique. Loin de l'apprentissage mécanique de l'empathie, l'écriture d'une rencontre avec un patient pousse l'étudiant à appréhender ce moment dans sa globalité. Elle le confronte à la variété des contextes culturels et sociaux qui per- mettent d'éclairer cette rencontre, en se servant de ce formidable outil qu'est le langage. Le langage reste un parent pauvre de nos études médicales. Pourtant, n'estil pas vrai que nous observons aussi avec des mots? L'écriture ouvre donc un espace de réflexion. Elle permet aux étudiants de se situer par rapport aux connaissances apprises. Elle leur fait expérimenter les limites

\section{Par l'écriture, l'étudiant appréhende la com-}

plexité de la rencontre avec un patient en opérant, par le biais de la narration, une distanciation et un recul réflexif.

et les difficultés d'expression qui peuvent entraver la relation thérapeutique, mais elle leur révèle aussi la richesse de ressources langagières trop souvent ignorées.

Après une double lecture des 115 textes reçus cette année, les 3 meilleurs textes francophones ont été anonymisés et envoyés à un jury composé de Mme Isabelle Chassot (Directrice de l'Office fédéral de la culture), Mme Pia Coppex (Présidente de la Société Suisse d'Ethique Biomédicale) et M. Bertrand Kiefer (Rédacteur en chef de la Revue Médicale Suisse).

Al'issue de cette procédure, nous avons le plaisir de vous présenter ici le texte récompensé par le Prix Joseph et Marie-Anne Piller, à savoir "Le Bastion enneigé» de M. Mick Rosset. Les jurés ont notamment apprécié dans ce texte un scénario bien construit, qui insiste sur l'isolement social des personnes âgées et un certain mal de vivre auquel le médecin est confronté. L'écriture rythmée, les descriptions vivantes et les observations précises permettent au lecteur de visualiser clairement le déroulement de la visite.

\section{Remarque}

La livraison germanophone du Bulletin des médecins suisses publie cette semaine le texte de M. Benedikt Locher, lauréat du meilleur texte en allemand. 